\title{
El perfil del profesor de español como lengua extranjera: dimensión formativa, dimensión técnica y dimensión afectivo-emocional ${ }^{1}$
}

\author{
Spanish as a Foreign Language Teacher's Profile: \\ Formative Dimension, Technical Dimension \\ and Affective-Emotional Dimension
}

\author{
Isabel Iglesias Casal \\ Spanish Philology Department, University of Oviedo, \\ c/ Teniente Alfonso Martínez, s/n, 33011 Oviedo, Spain \\ e-mail: iscasal@uniovi.es
}

\begin{abstract}
In this article we will reflect on the three dimensions that constitute key areas in the professional development of teachers of Spanish as a foreign language (ELE): the formative dimension, the technical dimension and affective-emotional dimension. We focus on the context of educational practice, an area from which we can invite reflection, to analyze the beliefs of teachers or change the direction of their work in the classroom.
\end{abstract}

Keywords: Spanish as a foreign language, teacher training processes, teachers' competence, attitudes, values and emotional factors

\section{INTRODUCCIÓN}

Resulta innegable que las exigencias de los nuevos tiempos requieren el desarrollo de nuevas habilidades que permitan adaptarse a un mundo en permanente

\footnotetext{
${ }^{1}$ Este trabajo se ha realizado con la ayuda obtenida para apoyar actividades de grupos de investigación de Arte, Humanidades y de Ciencias Sociales y Jurídicas de la Universidad de Oviedo, financiada por la Consejería de Educación, Cultura y Deporte del Principado de Asturias [Cód. 201510863].
} 
cambio, un mundo donde el conocimiento se manifiesta de muy diversas maneras y está sometido a un proceso continuo de transformación. Y es en esta realidad cambiante donde la formación del profesorado de lenguas extranjeras cobra especial relevancia porque debe preparar para los retos que nos plantea una sociedad dinámica y abierta que nos lleva a los docentes de manera ineludible a reflexionar sobre nuestro perfil profesional en escenarios caracterizados por continuos avances tecnológicos o reestructuraciones sociales y culturales que nos demandan una constante actualización.

Como afirman Richard Bowe, Stephen J. Ball \& Anne Gold (1992, en Esteve Zaragaza, 2006, p. 23) el cambio educativo se genera simultáneamente en tres contextos diferentes: el contexto macro, que depende de la evolución de las fuerzas sociales, los grupos políticos y los sectores económicos y financieros; el contexto político y administrativo, que pretende ordenar la realidad mediante leyes y decretos que siempre tienen una capacidad de cambio limitada; y finalmente, el contexto de la práctica educativa, constituido por el trabajo diario de los docentes en el aula y el funcionamiento real de los centros de enseñanza.

En este artículo nos centraremos en el contexto de la práctica educativa, ámbito desde el que podemos invitar a la reflexión, analizar las creencias del profesorado o modificar el sentido de su trabajo en las aulas. Partiremos de los datos obtenidos en una investigación que llevamos a cabo en la Universidad de Oviedo (Iglesias Casal, 2016) sobre la figura del profesor ideal de lenguas extranjeras, en la que establecimos una comparativa de perfiles desde el paradigma experto versus novel ${ }^{2}$. Este trabajo, en el que adoptamos el enfoque biográfico-narrativo, recogía algunos de los resultados de un estudio realizado para conocer el perfil competencial de un buen docente, mediante la identificación y descripción de las cualidades que estudiantes de posgrado atribuyen a los profesores excelentes. En el presente artículo reflexionaremos sobre las tres dimensiones que emergieron de los datos facilitados por nuestros informantes en el mencionado estudio y que, en nuestra opinión, configuran ámbitos clave en el desarrollo profesional de los docentes de ELE: la dimensión formativa, la dimensión técnica y la dimensión afectivo-emocional.

Jacques Delors en el informe La educación encierra un tesoro (1996) defiende una perspectiva de la educación a lo largo de la vida que debe basarse en cuatro pilares: aprender a conocer, aprender a hacer, aprender a vivir y aprender a ser. Podríamos establecer conexiones evidentes entre estos cuatro pilares y las tres dimensiones que nos van a servir para vertebrar nuestra reflexión sobre el perfil competencial del profesorado de ELE, tal como queda reflejado en el siguiente diagrama:

\footnotetext{
${ }^{2}$ Esta investigación constituye la primera fase de un estudio más amplio que se está desarrollando en el Grupo ALCE (Aprendizaje de Lenguas y Culturas Extranjeras) y fue presentado en el XXVI Congreso Internacional de ASELE: La formación y competencias del profesorado de ELE, celebrado en septiembre de 2015 en el Centro de Lenguas Modernas de la Universidad de Granada.
} 


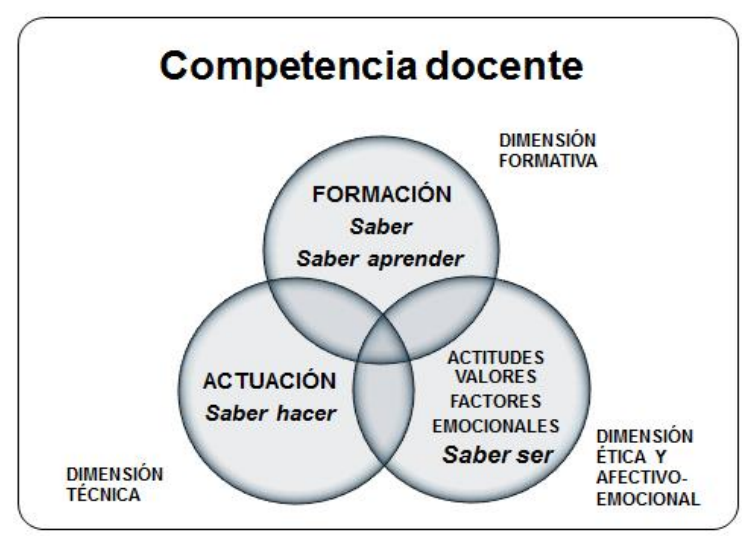

Fig. 1. La triple dimensión en el análisis de la competencia docente

Como podemos observar, estos tres ámbitos presentan fronteras permeables que se interrelacionan y juntos colaboran sinérgicamente en la construcción de la competencia docente.

\section{DIMENSIÓN FORMATIVA: SABER Y SABER APRENDER}

Si partimos de una concepción holística de la dimensión formativa, las instituciones y los profesionales que tenemos alguna responsabilidad en la gestión de los currículos y de los planes docentes de estudios de grado o de posgrado para profesorado de ELE debemos ser conscientes del error que puede suponer dar prioridad casi absoluta a la adquisición de conocimientos en detrimento de otros ámbitos que constituyen también elementos esenciales en la construcción de una competencia docente equilibrada: la formación didáctica y pedagógica del profesorado.

En la mencionada investigación que llevamos a cabo en la Universidad de Oviedo (Iglesias Casal, 2016) se analizaron las representaciones de los informantes aplicando como estrategia de recogida de datos un instrumento basado en una técnica indirecta y no interactiva: el análisis documental. Formulamos a 53 estudiantes de posgrado una pregunta abierta, descriptiva, no directiva: En tu opinión ¿qué cualidades crees que constituyen el perfil del profesor ideal de ELE? Elabora un decálogo y describe brevemente esas características. Ello nos permitió captar su punto de vista sin la interferencia del investigador y dar cuenta del modo en que los informantes conciben y asignan contenido al concepto "profesor ideal"; en esto reside, precisamente, la significatividad y confiabilidad de la información porque son los propios informantes quienes introducen sus prioridades.

$\mathrm{Al}$ analizar y describir las creencias percibidas por los informantes, en la configuración de la excelencia docente se muestra un ineludible equilibrio entre el dominio de los 
contenidos y la formación didáctica y pedagógica. En el apartado de formación, emergieron en el estudio cuatro categorías que arrojaron los siguientes porcentajes de frecuencia:

Tabla 1. Categorías y porcentajes de la dimensión formativa

\begin{tabular}{|l|c|}
\hline \multicolumn{1}{|c|}{ Formación } & $\begin{array}{c}\text { Frecuencia } \\
{[\%]}\end{array}$ \\
\hline 1. Formación académica (Contenidos vinculados a disciplina) & 96,22 \\
\hline 2. Formación didáctica y pedagógica & 90,5 \\
\hline 3. Compromiso de formación continua & 37,73 \\
\hline 4. Competencia digital & 11,32 \\
\hline
\end{tabular}

\subsection{FORMACIÓN ACADÉMICA Y FORMACIÓN DIDÁCTICO-PEDAGÓGICA}

Gran parte del profesorado piensa todavía que un buen dominio de los contenidos disciplinares exime en buena medida de saberes pedagógicos o didácticos. Sin embargo, como hemos visto en el gráfico anterior, los porcentajes correspondientes a la formación académica y a la formación didáctica y pedagógica alcanzan puntuaciones similares. Recordemos que nuestros informantes son estudiantes de posgrado, que se encuentran en lo que hemos llamado "etapa bisagra", a caballo entre dos fases: la de estudiante y la de profesor novel. No resulta extraño, pues, que consideren como una unidad dialéctica el saber teórico (la transmisión de contenidos) y la praxis pedagógica (que supone el conocimiento de los procesos de aprendizaje), es decir, que señalen la necesidad de una "transposición didáctica del saber sabio al saber enseñado", en palabras de Y. Chevallard (1991).

Sin embargo, pese al deseado equilibrio entre el dominio cognoscitivo de lo que se va a enseñar y las necesarias habilidades de comunicabilidad didáctica, P. Perrenoud (2001) afirma que "la formación de docentes es, sin duda, una de las menos provistas de observaciones empiricas metódicas sobre las prácticas, sobre el trabajo real de los profesores, en lo cotidiano y en su diversidad" (p. 509). Justamente para hacer evolucionar las prácticas, este sociólogo suizo defiende la necesidad de describir las condiciones y las dificultades reales a las que se enfrenta diariamente el profesorado:

Esto se complica porque numerosos curricula de formación inicial se fundan en una visión prescriptiva del oficio antes que en un análisis preciso de su realidad. Por supuesto, nada obliga a conformar la formación inicial a la realidad actual de un oficio, en todos sus aspectos. La formación no tiene ninguna razón de estar completamente al lado de la reproducción, ella debe anticipar las transformaciones. (p. 509) 
Perrenoud (2001) propone diez criterios ${ }^{3}$ a los que, en su opinión, debería responder una buena formación profesional de los docentes. Aunque deja claro que no defiende una perspectiva excesivamente utilitaria de los saberes teóricos, critica abiertamente la acumulación de contenidos "que se justifican solo por la tradición, por un argumento de autoridad o por la influencia de un grupo de presión". Aboga por una formación crítica y reflexiva en la que teoría y práctica se articulen de manera equilibrada.

Estamos plenamente de acuerdo con esta visión holística de los procesos formativos, por eso, para evitar esta fractura, deberíamos apelar a la corresponsabilidad de formadores académicos y tutores de las prácticas profesionales: unos y otros debemos ser conscientes de que colaboramos de forma mancomunada en la consecución de los mismos objetivos y en la construcción y desarrollo de las mismas competencias. Para ello debemos favorecer y reforzar el trabajo en equipo de los formadores (teóricos y prácticos) facilitando el desarrollo de competencias vinculadas a la observación y al análisis del trabajo llevado a cabo en situaciones concretas. Esta coordinación conllevaría -por parte de los gestores académicos de las instituciones $\mathrm{y}$, en último término, de los gestores políticos- no solo coordinar los períodos de prácticas profesionales, sino también perfilar qué papel han de desempeñar los tutores, qué tareas y qué responsabilidades se les asignan.

Algo que llevamos reclamando algunos responsables de másteres universitarios en español como lengua extranjera es que se retribuya económicamente a los tutores de prácticas o que, al menos, que el esfuerzo y la dedicación que invierten sean reconocidos como horas de su carga docente. Asimismo, en nuestra opinión, los contenidos teóricos se movilizan y se transfieren de manera más fluida si los docentes sobre los que recae la tarea de la formación "teórica" tienen una amplia experiencia sobre el terreno y han tenido la oportunidad de teorizar sobre las prácticas y de desarrollar prácticas reflexivas.

Si nos circunscribimos al ámbito de la enseñanza de español como lengua extranjera, podríamos preguntarnos si la formación actual del profesorado capacita a los docentes para enfrentarse con cierta garantía de éxito a las condiciones cambiantes de una sociedad cada vez más plural y más innovadora. Precisamente el XIX Congreso Internacional de ASELE celebrado en Cáceres en 2008 se centró en la figura del profesor de español LE/L2. En una de las mesas redondas se debatió sobre

\footnotetext{
${ }^{3}$ Los diez criterios propuestos son los siguientes: una transposición didáctica fundada en el análisis de las prácticas y de sus transformaciones; un referencial de competencias que identifican los saberes y capacidades requeridos; un plan de formación organizado en torno a competencias; un aprendizaje a través de problemas, un procedimiento clínico; una verdadera articulación entre teoría y práctica; una organización modular y diferenciada; una evaluación formativa fundada en el análisis del trabajo; tiempos y dispositivos de integración y de movilización de los saberes; una asociación negociada con los profesionales; un corte favorable de los saberes para su movilización en el trabajo.
} 
el tipo de formación que debería tener un buen profesor de español como lengua extranjera. M. ${ }^{a}$ Luz Gutiérrez Araus centró sus reflexiones en cinco puntos: 1) formación de profesores nativos/no nativos; 2) nivel de formación del que parte el futuro profesor; 3) formación inicial, formación de posgrado y formación permanente; 4) contenidos de esta formación; 5) tipo de formación: presencial/no presencial.

En relación con el nivel de formación del que parte el futuro profesor de ELE, como bien señala la profesora Gutiérrez Araus (2009, pp. 80-81), los titulados que demandan formarse como profesores de español L2 provienen de licenciaturas y de grados de muy diversos ámbitos de conocimiento, por lo que no todos parten de la preparación lingüística de un filólogo. Gutiérrez Araus afirma que, aunque en estos casos podrían establecerse dos itinerarios diferentes en los currículos, cuando esto no es posible, una opción adecuada para atajar esta disfunción de base consistiría en establecer tutorías personalizadas para estos alumnos, de este modo se detectarían las posibles carencias y se ayudaría a suplirlas.

La profesora Gutiérrez Araus (2009, pp. 83-84) formula una propuesta que concita en la actualidad un amplio consenso en cuanto a los contenidos generales vertebradores de los cursos de formación. Estos deberían incluir un primer grupo de materias sobre los fundamentos teóricos de la enseñanza del español como segunda lengua (conceptos básicos de la lingüística aplicada a la enseñanza de segundas lenguas y metodología en la enseñanza/aprendizaje de segundas lenguas). El segundo grupo abarcaría aquellas materias que abordan una descripción lingüística explícita del español según los niveles habituales para la sistematización de los fenómenos de la lengua: el componente fonético-fonológico; aspectos gramaticales y léxicos-semánticos; el componente discursivo y pragmático (géneros discursivos y tipos de textos), sin olvidar nunca que la perspectiva para abordar la materia ha de ser siempre la del español como lengua extranjera. El tercer grupo se centraría en el estudio de las variedades diatópicas y diastráticas en los usos del español.

Tampoco deberían olvidarse otras materias como las tecnologías de la información y de la comunicación (TIC), sociedad y cultura española e hispánica (incluidas también la literatura, la historia y el arte), el español para fines específicos, el desarrollo de la competencia comunicativa intercultural, o la enseñanza de español a inmigrantes, tanto en contexto escolar como a colectivos de adultos alfabetizados o sin alfabetizar.

Finalmente, la planificación de los procesos formativos debería surgir de las exigencias y necesidades concretas derivadas del ejercicio profesional. Por este motivo, antes de la realización del prácticum se podrían planificar en el aula prácticas debidamente tutorizadas que constituirían verdaderas experiencias de aprendizaje para los alumnos, experiencias a través de las cuales se dotaría a los futuros docentes de las herramientas necesarias para solucionar los problemas a los que se tienen que enfrentar en el ejercicio de su profesión. 


\subsection{COMPROMISO DE FORMACIÓN CONTINUA}

Aunque la formación inicial representa un papel relevante como una primera e indispensable fase, el proceso formativo del profesorado debería entenderse como un continuum. La idea del lifelong learning ${ }^{4}$ implica la necesidad de actualización permanente para poder ofrecer respuestas adecuadas en el marco de nuestro ejercicio profesional. Por tanto, debería formar parte del código deontológico de quienes nos dedicamos a enseñar y a formar a formadores.

Partiendo de la concepción del docente como sujeto activo de su formación, Montero Mesa (2006) subraya que la meta irrenunciable de las diversas actuaciones de la formación ha de ser el desarrollo profesional de quienes participan en ella:

A partir de la consideración de profesores y profesoras como profesionales de la enseñanza, y de la formación como un proceso de socialización y desarrollo -personal y profesional- a través de los tiempos y espacios que los profesores recorren durante su preparación y su ejercicio profesional, las ideas de actividad, aprendizaje continuo y reconstrucción del conocimiento profesional son ejes potencialmente estructuradores de la formación y claves para interpretar sus procesos y resultados. (p. 70)

Así pues, la formación continua del profesorado de español tanto nativo como no nativo y la actualización del currículo académico, deben constituir la piedra angular de la mejora de la profesión docente. A esta mejora puede contribuir, sin duda, la oferta de cursos para la actualización de profesores organizados por los distintos centros del Instituto Cervantes y la participación en congresos, jornadas, seminarios o encuentros prácticos que cada año se ofertan desde las universidades y desde otras instituciones. De igual modo, la difusión de revistas electrónicas y publicaciones especializadas propician la actualización de profesores.

Zabalza Beraza (2006, pp. 51-57) propone cuatro consideraciones, a nuestro juicio muy relevantes, que tendrían que constituir la nueva hoja de ruta en la formación del profesorado y que, en nuestra opinión deberíamos contemplar también en el ámbito del ELE como verdaderos compromisos de formación continua: a) plantear la formación como un proceso a largo plazo; b) revisar la figura de profesor que sirve de referente a la formación; c) reforzar el sentido profesionalizador de la formación; d) dar un nuevo enfoque al aprendizaje: de la formación dirigida a la asimilación

\footnotetext{
${ }^{4}$ El capítulo quinto del Informe a la UNESCO de la Comisión Internacional sobre la Educación para el Siglo XXI (La educación encierra un tesoro, 1996) está dedicado a la educación a lo largo de la vida, un concepto que supera la tradicional distinción entre educación primera y educación permanente. Desde esta perspectiva, la sociedad se constituye en una fuente de aprendizaje y de desarrollo de las capacidades del individuo. Esta idea de educación como una experiencia social, pluridimensional y escalonada durante toda la vida, "permite, ordenar las distintas etapas, preparar las transiciones, diversificar y valorizar las trayectorias".
} 
o acumulación de conocimientos ${ }^{5}$ a la formación dirigida a la acomodación y construcción de saberes prácticos.

Asimismo, es preciso conectar de manera ineludible la formación inicial y la formación continua, pues como señalaba Andy Hargreaves (en Zabalza Beraza, 2006), "ningún sistema de formación inicial puede conseguir el impacto que logra la formación continua" (p. 53), porque países con una formación inicial débil pero con una formación continua fuerte alcanzan niveles más altos de calidad en la enseñanza que en aquellos casos en los que se da la combinación contraria.

Como hemos visto en este apartado, los retos educativos que afrontamos en las sociedades del siglo XXI no pueden hacernos olvidar que estamos obligados a formar a profesores de lenguas extranjeras altamente cualificados y comprometidos con su labor. Sin ese compromiso, la tarea docente corre el riesgo de reducirse únicamente a brindar enseñanzas, olvidándose de facilitar aprendizajes.

\subsection{COMPETENCIA DIGITAL}

Todos los países europeos han desarrollado políticas que establecen directrices específicas para favorecer la implementación del uso de las TIC con fines pedagógicos. El 18 de febrero de 2014 se presentó en España el primer borrador del proyecto de Marco Común de Competencia Digital Docente, cuyos objetivos eran, entre otros, facilitar una referencia común a profesores y formadores, disponer de una lista estatal de competencias mínimas de los profesores, contribuir al cambio metodológico y hacer posible que los profesores faciliten y evalúen el desarrollo de la competencia digital de sus alumnos.

Se acordó empezar a trabajar en la creación de una herramienta que pudiera facilitar tres tipos de evaluación: 1) auto-evaluación individual que permita diagnosticar necesidades formativas; 2) pruebas de evaluación por niveles, con la posibilidad de combinar las pruebas con un portfolio profesional de competencia digital con unos criterios comunes de evaluación; 3) evaluación de centros educativos que incluyan en el proyecto educativo del centro también otros criterios relacionados con el uso de recursos y entornos digitales ${ }^{6}$.

\footnotetext{
${ }^{5}$ Zabalza utiliza los dos conceptos piagetianos de "asimilación" (incorporación de nuevas informaciones a nuestros esquemas) y "acomodación" (la información nueva modifica y reestructura nuestros esquemas previos). Como se sabe, el verdadero aprendizaje se vincula a la reestructuración de esquemas previos, tanto conceptuales como operativos, sobre la base de informaciones y experiencias nuevas.

${ }^{6}$ Con esta lista de objetivos clara desde un principio, se establecen entonces tres líneas de actuación en las que el Instituto Nacional de Tecnologías Educativas y de Formación del Profesorado (INTEF), del Ministerio de Educación, Cultura y Deporte, ha estado trabajando durante los últimos cuatro años: a) Propuesta de marco común de referencia; b) Plan de evaluación y acreditación de profesores y de centros; c) Impulso en paralelo a la formación docente en competencia digital realizando convocatorias anuales de cursos de Formación en Red para profesores no universitarios.
} 
No obstante, sorprende que en nuestra investigación (Iglesias Casal, 2016) solo un $11,32 \%$ de los informantes hayan señalado la importancia de la competencia digital docente, pues -tal como recoge el Marco Común de Competencia Digital Docenteesta constituye una de las áreas de la profesionalización peor atendidas en la formación inicial, pese a que, sin duda, facilita un aprendizaje más eficaz, motivador e inclusivo. Además, se ha demostrado que el uso adecuado de las TIC incrementa la motivación de los estudiantes, al proporcionarles un control más personal e individualizado sobre su propia experiencia de aprendizaje, lo que puede afectar positivamente al rendimiento académico.

Desde el año 2007, el uso de las TIC en el ámbito educativo constituye uno de los ejes transversales del programa de aprendizaje permanente y se ha convertido en una prioridad de los programas europeos Comenius, Erasmus, Leonardo da Vinci $y$ Grundtvig. La Comisión Europea también insiste en el potencial que suponen las TIC en el fomento de innovaciones metodológicas y las considera esenciales para perfeccionar las competencias del siglo XXI, lo que, evidentemente, implica que se integre en el currículo de la formación del profesorado el desarrollo cada vez más amplio de sus aplicaciones pedagógicas.

\section{DIMENSIÓN TÉCNICA: ACTUACIÓN (SABER HACER)}

Sin duda alguna, el paso de una docencia centrada en la enseñanza a una enseñanza centrada en el aprendizaje pone a prueba la solvencia para afrontar situaciones didácticas complejas en las que se miden las habilidades y destrezas técnicas desarrolladas en la organización y gestión del proceso de enseñanza/aprendizaje.

Por este motivo, para Perrenoud (2001) la formación del profesorado tendría que orientarse hacia un aprendizaje a través de casos, organizándose en torno a situaciones singulares que favorezcan la movilización de conocimientos previos y que ayuden a construir nuevos saberes de formación. Parece evidente que un ejercicio profesional eficaz exige una formación centrada en el desarrollo práctico de procesos sólidamente fundamentados en la teoría. Sin embargo, las investigaciones en torno a la transferencia de conocimientos concluyen que esa movilización de saberes no se adquiere automáticamente, sino que es preciso abordarla como parte esencial de los currículos en los procesos formativos del profesorado.

La etapa puente entre la formación inicial y la permanente se conoce como inducción y constituye la base para el desarrollo profesional continuo. Bolam (1995) define el período de inducción como "el proceso de apoyo y de formación que de forma creciente se ve necesario para el éxito en el primer año de enseñanza" (p. 613). En una línea de pensamiento similar, Bozu (2010), en su estudio sobre el proceso de inducción o socialización profesional de los profesores universitarios noveles, afirma que "el ajuste y la adaptación a esta nueva realidad suelen ir acompañados de lo que se denomina aprendizaje de autorreflexión, un tipo de aprendizaje 
dirigido al cambio y al autodesarrollo, en lugar del prescriptivo y estructurado de la universidad" (p. 63), donde se priorizan los contenidos conceptuales sobre los contenidos procedimentales y actitudinales, frente a lo que ocurre en el ámbito profesional, donde prevalecen los procedimientos y las actitudes sobre los contenidos. Para muchos autores, este proceso de inducción se vincula a la socialización profesional de los docentes y a lo que ellos mismos denominan "choque con la realidad" originado cuando se confrontan las expectativas y experiencias reales de trabajo con las que los profesores generaron cuando se estaban formando en la universidad.

Perrenoud (2001) insiste en que no puede formarse a un práctico reflexivo imponiéndole formas ortodoxas para impartir clase. Y es bien cierto porque, como sabemos, ninguna práctica compleja puede simplificarse aplicando, sin más, un determinado saber. En nuestra opinión, el encaje y la adaptación a la nueva realidad exigen que "el conocimiento sobre la práctica (in vitro)" se movilice o se transfiera al contexto de una verdadera praxis (in vivo, in situ). Y esa transferencia supone aplicar en contexto cuantas habilidades y destrezas se hayan adquirido relacionadas, entre otros, con ámbitos como la planificación, los objetivos, la metodología, las necesidades en intereses de los estudiantes, el diseño, la selección y la explotación de materiales, el tratamiento del error o el proceso de evaluación.

Cobra, así, relevancia el concepto de práctica reflexiva, pues como defiende Perrenoud, reflexionar sobre la experiencia favorece la construcción de nuevos saberes. Erazo-Jiménez (2009) concibe la práctica reflexiva como la piedra angular del desarrollo profesional docente porque representa una metacompetencia que constituye la base sobre la que se actualizan, profundizan y movilizan los conocimientos profesionales. Por tanto, desarrollar esa práctica reflexiva en contextos reales de interacción supone una vía estratégica para fortalecer la calidad de la educación.

Bain (2005, p. 24), director del Center for Teaching Excellence de la Universidad de Nueva York, se dedicó durante años a estudiar el modus operandi de los mejores profesores de los Estados Unidos y concluye que cualquier estudio sobre la calidad docente debe recabar información sobre en qué medida el profesor ha ayudado a aprender a sus estudiantes y en qué medida ha estimulado su interés y su desarrollo intelectual. Afirma que, sin excepción, los profesores extraordinarios conocen su materia extremadamente bien, "saben cómo clarificar y simplificar conceptos complejos y son capaces de pensar sobre su propia forma de razonar en la disciplina, analizando su naturaleza y evaluando su calidad" (p. 27). Es decir, su capacidad de pensamiento metacognitivo facilita el control activo de los procesos mentales desarrollados durante el aprendizaje.

En el campo de la enseñanza de lenguas extranjeras, Estaire \& Fernández (2013) asumen un concepto de competencia docente que integra el "saber", el "saber ser", el "saber hacer" y el "saber aprender", y ofrecen un marco estimulante para diseñar

\footnotetext{
${ }^{7}$ Veenman (1984) popularizó el concepto de "shock de la realidad", aunque también se emplean otros términos como "shock de la transición" o "shock de la praxis".
} 
posibles vías de formación del profesorado. Parten de un enfoque centrado fundamentalmente en el "saber hacer" del docente, enfoque en el que las llamadas acciones didácticas constituyen el eje sobre el que se articulan los conocimientos que permiten al profesor llevar acabo esas acciones de manera consistente. Esos conocimientos proporcionan las bases para establecer los criterios de evaluación y autoevaluación y para desarrollar las acciones docentes dirigidas a la planificación didáctica o a la gestión y organización del aula. Las acciones didácticas entendidas de este modo también se encaminarán al desarrollo de estrategias, tanto comunicativas como de aprendizaje, sin olvidar la atención a los aspectos contextuales y a las posibles implicaciones socioculturales.

También en el ámbito del español, el Instituto Cervantes, elaboró en 2011 un exhaustivo informe titulado ¿Qué es ser un buen profesor o una buena profesora de $E L E$ ?, en el que se analizaron las creencias del alumnado, profesorado y personal técnico y directivo de la institución. Partiendo de los datos recabados en ese informe, publicó un año después Las competencias clave del profesorado de lenguas segundas y extranjeras (2012), que recoge ocho competencias clave de las que se derivan una serie de competencias específicas:

Tabla 2. Competencias del profesorado de LE/L2 (Fuente: Instituto Cervantes, 2012, p. 11)

\begin{tabular}{|c|c|}
\hline Competencias clave & Competencias específicas \\
\hline a) Organizar situaciones de aprendizaje & $\begin{array}{l}\text { - Promover el uso y la reflexión sobre la lengua. } \\
\text { - Planificar secuencias didácticas. } \\
\text { - Gestionar el aula. }\end{array}$ \\
\hline $\begin{array}{l}\text { b) Evaluar el aprendizaje y la actuación } \\
\text { del alumno }\end{array}$ & $\begin{array}{l}\text { - Servirse de herramientas y procedimientos de evaluación. } \\
\text { - Garantizar buenas prácticas en la evaluación. } \\
\text { - Promover una retroalimentación constructiva. } \\
\text { - Implicar al alumno en la evaluación. }\end{array}$ \\
\hline $\begin{array}{l}\text { c) Implicar a los alumnos en el control } \\
\text { de su propio aprendizaje }\end{array}$ & $\begin{array}{l}\text { - Promover que el alumno gestione los recursos y medios } \\
\text { disponibles para aprender. } \\
\text { - Integrar en la enseñanza herramientas para reflexionar } \\
\text { sobre el proceso de aprendizaje. } \\
\text { - Promover que el alumno defina su propio proyecto de } \\
\text { aprendizaje. } \\
\text { - Motivar al alumno para que se responsabilice de su propio } \\
\text { aprendizaje. }\end{array}$ \\
\hline d) Facilitar la comunicación intercultural & $\begin{array}{l}\text { - Implicarse en el desarrollo de la propia competencia } \\
\text { intercultural. } \\
\text { - Adaptarse a las culturas del entorno. } \\
\text { - Fomentar el diálogo intercultural. } \\
\text { - Promover que el alumno desarrolle su competencia inter- } \\
\text { cultural. }\end{array}$ \\
\hline
\end{tabular}


tab. 2

\begin{tabular}{|c|c|}
\hline Competencias clave & Competencias específicas \\
\hline $\begin{array}{l}\text { e) Desarrollarse profesionalmente como } \\
\text { profesor de la institución }\end{array}$ & $\begin{array}{l}\text { - Analizar y reflexionar sobre la práctica docente. } \\
\text { - Definir un plan personal de formación continua. } \\
\text { - Implicarse en el desarrollo profesional del equipo docente. } \\
\text { - Participar activamente en el desarrollo de la profesión. }\end{array}$ \\
\hline $\begin{array}{l}\text { f) Gestionar sentimientos y emociones } \\
\text { en el desempeño de su trabajo }\end{array}$ & $\begin{array}{l}\text { - Gestionar las propias emociones. } \\
\text { - Motivarse en el trabajo. } \\
\text { - Desarrollar las relaciones interpersonales. } \\
\text { - Implicarse en el desarrollo de la inteligencia emocional } \\
\text { del alumno. }\end{array}$ \\
\hline $\begin{array}{l}\text { g) Participar activamente en la institu- } \\
\text { ción }\end{array}$ & $\begin{array}{l}\text { - Trabajar en equipo en el centro. } \\
\text { - Implicarse en los proyectos de mejora del centro. } \\
\text { - Promover y difundir buenas prácticas en la institución. } \\
\text { - Conocer la institución e integrarse en ella. }\end{array}$ \\
\hline $\begin{array}{l}\text { h) Servirse de las TIC para el desem- } \\
\text { peño de su trabajo }\end{array}$ & $\begin{array}{l}\text { - Implicarse en el desarrollo de la propia competencia digital. } \\
\text { - Desenvolverse en entornos digitales y con aplicaciones } \\
\text { informáticas disponibles. } \\
\text { - Aprovechar el potencial didáctico de las TIC. } \\
\text { - Promover que el alumno se sirva de las TIC para su } \\
\text { aprendizaje. }\end{array}$ \\
\hline
\end{tabular}

Este documento del Instituto Cervantes sobre las competencias clave constituye, sin duda, un modelo marco para el desarrollo coherente de acciones formativas dirigidas a los docentes y se ha adoptado ya como referente en numerosas instituciones académicas para definir perfiles docentes, implementar y desarrollar procedimientos de evaluación del desempeño, o aplicar criterios en la selección y promoción del profesorado.

Podríamos preguntarnos por la utilidad de estos referenciales de competencias que constituyen para algunos instrumentos de control, por lo que niegan la pertinencia de describir de forma metódica las competencias profesionales. Perrenoud (2004, p. 154) argumenta que su función principal consiste en ayudar a formular y a estabilizar una visión clara del oficio, porque no deben entenderse "como instrumentos reservados a los expertos, sino medios para que los profesionales puedan construir una identidad colectiva". No parece desacertado, pues, organizar los planes de formación en torno a competencias que deben empezar a trabajarse desde la formación inicial, si bien el debate sobre un referencial obliga, según Perrenoud, a evaluar la diversidad de los puntos de vista y los límites del consenso. Defiende la idea de que su formulación sea provisional para que el debate prosiga. En este sentido, estamos plenamente de acuerdo, el debate sobre estos referenciales de competencias es más importante que el consenso. 


\section{DIMENSIÓN AFECTIVO-EMOCIONAL: ACTITUDES, VALORES Y FACTORES EMOCIONALES (SABER SER)}

Jane Arnold y H. Douglas Brown afirman que desde el punto de vista del aprendizaje afectivo de lenguas extranjeras, ser es tan importante como hacer, porque "un buen profesor de idiomas sabe y hace, pero esencialmente es" (en Arnold, 2000, p. 22). Esto significa que nuestra labor resultará mucho más eficaz si nos preocupamos también de nuestra propia inteligencia emocional, que indudablemente aporta innumerables ventajas al proceso de aprendizaje a la hora de desarrollar en los alumnos destrezas y capacidades tanto cognoscitivas como afectivas.

Aludiendo al estrecho vínculo establecido entre los dos componentes de la formación del profesorado: los sujetos y las medidas (contenidos, estrategias, recursos, formadores...), Montero Mesa (2006, p. 70) recuerda la advertencia realizada por Gilles Ferry (1983): "más formación no implica necesariamente mejores profesionales". Afirmaciones como esta subrayan el papel relevante que cada profesor, como sujeto individual, desempeña en la construcción de su propio perfil como docente.

Asumiendo esta premisa, en un trabajo anterior (Iglesias Casal, 2014) abordamos el estudio de las competencias generales y de las competencias docentes del profesorado de ELE en el s. XXI estableciendo para ello dos ámbitos -el profesional y el personal-. En el primero reflexionamos sobre los perfiles investigador y docente; en el segundo analizamos al profesor como sujeto social, con sus rasgos de personalidad y su manera de gestionar la dimensión afectiva.

En la investigación posterior que llevamos a cabo con estudiantes del máster en ELE de la Universidad de Oviedo (Iglesias Casal, 2016) quedó claramente demostrado que las creencias de los estudiantes de posgrado sobre el profesor ideal no reflejan únicamente características relacionadas con su formación o con las técnicas pedagógicas que utiliza en la gestión del aula o en la mejora del proceso de enseñanza/aprendizaje, sino también -y de manera muy relevante- con su entidad como persona, y por tanto, con sus actitudes, valores y factores emocionales. Como se puede apreciar en la siguiente tabla, nuestros informantes destacan la relevancia de las características personales del docente (45\%), por encima de las competencias académicas y profesionales que se enmarcan dentro de los ámbitos de formación y de actuación:

Tabla 3. Creencias de estudiantes de posgrado sobre el perfil del profesor ideal de ELE: valor porcentual de los ámbitos de formación, actuación y actitudes (Fuente: Investigación Máster en ELE Universidad de Oviedo, 2016)

\begin{tabular}{|l|c|c|}
\hline \multicolumn{1}{|c|}{ Ámbito } & Frecuencia de aparición & Porcentaje \\
\hline Formación & 125 & 20,3 \\
\hline Actuación & 214 & 34,7 \\
\hline Actitudes, valores y factores emocionales & 276 & 45 \\
\hline
\end{tabular}


Para nuestros 53 informantes, las diez características principales vinculadas con la dimensión ética (actitudes y valores) y afectivo-motivacional fueron las siguientes: amigable y cercano, accesible (abierto, amable, agradable) (69,8\%); empático (52,8\%); motivador (37,7\%); con sentido del humor (32\%); tolerante (22,6\%); actitud optimista y entusiasta (18,8\%); respetuoso (16,9\%); fomenta la interculturalidad y los valores sociales $(15,9 \%)$; transmite confianza y seguridad en sí mismo $(9,4 \%)$; es consciente de su labor como educador $(7,5 \%)$.

Recordemos que el desarrollo de las actitudes se lleva a cabo tanto en las situaciones de aprendizaje como en las relaciones sociales que se establecen en el aula; además los aspectos actitudinales ejercen un cierto control sobre el aprendizaje porque promueven procesos de colaboración y ayudan a regular los factores afectivos. En este sentido, los profesores de lenguas extranjeras deberíamos suscribir la Recomendación del Parlamento Europeo y del Consejo de 18 de diciembre de 2006 sobre las competencias clave para el aprendizaje permanente. En el apartado sobre Competencias sociales y cívicas -que incluyen las personales, interpersonales e interculturales- se recogen los tipos de comportamiento que preparan a los sujetos para resolver conflictos y participar eficazmente en los ámbitos sociales y profesionales que caracterizan una sociedad cada vez más diversa.

Los elementos fundamentales de esta competencia abarcan aspectos que podríamos incluir en esta dimensión afectivo-emocional para configurar el perfil del profesorado de ELE: la capacidad de ser tolerantes y empáticos, de comprender puntos de vista diferentes, de comunicarse de modo constructivo en entornos muy variados mostrando una alta capacidad de negociar sin generar desconfianza.

Asimismo, para desarrollar esas competencias sociales y cívicas debemos fomentar el interés por la comunicación intercultural y enfrentarnos al reto que nos plantea la diversidad de los sistemas de valores con los que nos relacionamos. Ahora bien, en nuestra opinión, la apreciación y la comprensión de las diferencias existentes entre los sistemas de valores de las distintas culturas, religiones o grupos étnicos no debe conducir a la pérdida de sentido crítico y admitir que cualquier situación o cualquier comportamiento pueden ser justificados apelando al argumento del relativismo cultural. El respeto pleno de los derechos humanos y de la igualdad es la base de una sociedad democrática y debería constituir uno de los objetivos prioritarios del encuentro entre culturas diferentes.

\section{CONCLUSIONES}

Gutiérrez Araus (2009, p. 85) advertía contra el riesgo de caer en dos planteamientos desenfocados en la formación de profesores de español L2: "un exceso de teoricismo en los contenidos del currículo o un exceso de pedagogismo". A nuestro modo de ver, los profesores de lenguas extranjeras deberíamos alcanzar el dominio, 
tanto intelectual como práctico de la transposición didáctica de los saberes que debemos enseñar.

En la investigación llevada a cabo por Bain (2005, p. 28) se concluye que los mejores profesores asumen también que el aprendizaje tiene poco sentido si no es capaz de producir una influencia duradera en el modo en que el estudiante piensa, siente o actúa. Aceptar esta premisa debe llevarnos a admitir que el conocimiento es construido, no recibido, y a entender que nuestros cerebros son unidades que no solo almacenan información, también la procesan, por eso en la modificación de los modelos mentales o en los procesos de enseñanza-aprendizaje las preguntas desempeñan un papel crucial y ayudan a construir conocimiento (Bain, 2005, pp. 37-42).

Por otro lado, no debemos olvidar que la enseñanza tiene un marcado carácter vocacional y, por tanto, nuestro ejercicio profesional no está determinado únicamente por los conocimientos, destrezas o habilidades que seamos capaces de alcanzar, sino también por aquellos factores de carácter individual que constituyen la llamada "competencia existencial" (saber ser). Estos factores se relacionan con las motivaciones, las actitudes, los valores, las creencias, los estilos cognitivos y los factores vinculados a la personalidad que (re)configuran nuestra identidad, pero que también influyen decididamente en nuestro perfil profesional.

Desarrollar actitudes positivas, cumplir con la ética de la profesión, trabajar en valores y pautas de comportamiento, constituye también un modelo de actuación para los docentes. Una concepción más abierta de nuestra labor debería llevarnos a trascender una visión puramente instrumental de la educación. Delors (1996) afirmaba que, "en cierto sentido, la educación se ve obligada a proporcionar las cartas náuticas de un mundo complejo y en perpetua agitación y, al mismo tiempo, la brújula para poder navegar por él". Si no queremos exponernos a un naufragio, los profesores debemos esforzarnos por atender y mejorar las tres dimensiones que hemos analizado: formativa, técnica y afectivo-emocional. Asimismo, tendremos que apelar a las autoridades educativas para que contribuyan a la mejora de la formación docente implementando medidas eficaces con la dotación presupuestaria suficiente. $\mathrm{Y}$ aunque aún reste mucho camino por recorrer, lo que importa es que estemos dispuestos a afrontar el reto que supone educar en un mundo cambiante donde necesariamente habrán de convivir las diferencias. 


\section{BIBLIOGRAFÍA}

Arnold, J. B. (2000). La dimensión afectiva en el aprendizaje de idiomas. Madrid: Cambridge University Press.

Bain, K. (2005). Lo que hacen los mejores profesores de universidad (trad. de Ó. Barberá). Valencia: Publicacions de la Universitat de València ( $1^{\mathrm{a}}$ ed. inglesa, 2004).

Bolam, R. (1995). Teacher Recruitment and Induction. En: L. Anderson (Ed.), International Encyclopaedia of Teaching and Teacher Education (pp. 612-615). Oxford: Pergamon.

Bozu, Z. (2010). El profesorado universitario novel: estudio teórico de su proceso de inducción o socialización profesional. Revista Electrónica de Investigación y Docencia (REID), 3, 55-72.

Chevallard, Y. (1991). La transposición didáctica. Del saber sabio al saber enseñado. Buenos Aires: AIQUE Grupo Editor.

Comisión Internacional sobre la Educación para el Siglo XXI (1996). La educación encierra un tesoro. Madrid: Santillana.

Erazo-Jiménez, M. ${ }^{a}$ S. (2009). Práctica reflexiva como estrategia de desarrollo profesional: presencia y estructura en reuniones docentes. Educación y Educadores, 12 (2), 47-74.

Estaire, S. \& Fernández, S. (2013). Competencia docente en lenguas extranjeras y formación de profesores: un enfoque de acción. Madrid: Edinumen.

Esteve Zaragaza, J. M. (2006). La profesión docente en Europa: perfil, tendencias y problemática. La formación inicial. Revista de Educación, 340, 19-40. Disponible en http://www.revista educacion.mec.es/re340/re340.pdf

Gutiérrez Araus, M. ${ }^{a}$ L. (2009). Nuevos tiempos y nuevos retos en la formación del profesor de español como segunda lengua. En: A. Barrientos Clavero (Coord.), El profesor de español LE-L2, Actas del XIX Congreso Internacional de la Asociación para la Enseñanza del Español como Lengua Extranjera (ASELE) (vol. 1, pp. 77-88). Cáceres: Servicio de Publicaciones de la Universidad de Extremadura.

Iglesias Casal, I. (2014). Competencias generales y competencias docentes del profesorado de ELE en el s. XXI: nuevas rutas, nuevos retos. Anuario brasileño de estudios hispánicos, 24, 57-72.

- (2016). El profesor ideal de lenguas extranjeras: una comparativa de perfiles desde el paradigma experto-novel. En: M. Á. Lamolda González (Comp.) \& O. Cruz Moya (Ed.), La formación y competencias del profesorado de ELE (pp. 493-503). Málaga: ASELE.

Instituto Cervantes (2011). ¿Qué es ser un buen profesor o una buena profesora de ELE? Análisis de las creencias del alumnado, profesorado y personal técnico y directivo de la institución. Disponible en http://cfp.cervantes.es/imagenes/File/recursos_proyectos/informe_buen_profesor_ ele/informe-buen-profesor-cervantes.pdf

- (2012). Las competencias clave del profesorado de lenguas segundas y extranjeras. Disponible en http://cvc.cervantes.es/ensenanza/biblioteca_ele/competencias/default.htm

Ministerio de Educación, Cultura y Deporte (2013). Marco Común de Competencia Digital Docente. Disponible en http://educalab.es/documents/10180/12809/MarcoComunCompeDigi DoceV2.pdf

Montero Mesa, M. ${ }^{a}$ L. (2006). Profesores y profesoras en un mundo cambiante: el papel clave de la formación inicial. Revista de educación, 340, 66-86. Disponible en http://www.revistaedu cacion.mec.es/re340/re340.pdf

Parlamento Europeo (2006). Recomendación del Parlamento Europeo y del Consejo de 18 de diciembre de 2006 sobre las competencias clave para el aprendizaje permanente. Disponible en https://www.boe.es/doue/2006/394/L00010-00018.pdf 
Perrenoud, P. (2001). La formación de los docentes en el siglo XXI. Revista de Tecnología Educativa, 14 (3), 503-523. Disponible en http://www.unige.ch/fapse/SSE/teachers/perrenoud/php_ main/php_2001/2001_36.html

- (2004). Diez nuevas competencias para enseñar: Invitación al viaje. Barcelona: Graó (Biblioteca de Aula, 196).

Zabalza Beraza, M. A. (2006). Buscando una nueva hoja de ruta en la formación del profesorado. Revista de Educación, 340, 51-58. Disponible en http://www.revistaeducacion.mec.es/re340 /re340.pdf 
